\title{
Molecular Mechanism of microRNA-155 in Connective Tissue Diseases
}

\author{
Jennifer L. Giguere, Jessica A. Mabragana \\ College of Health and Human Sciences, Northern Illinois University, USA.
}

\begin{abstract}
How to cite this paper: Jennifer L. Giguere, Jessica A. Mabragana. (2020) Molecular Mechanism of microRNA-155 in Connective Tissue Diseases. International Journal of Clinical and Experimental Medicine Research, 4(3), 41-46. DOI: 10.26855/ijcemr.2020.07.002
\end{abstract}

Received: April 22, 2020

Accepted: May 28, 2020

Published: June 24, 2020

\begin{abstract}
Abnormal expression of miRNAs is related to many diseases, including immune cells rheumatoid arthritis, systemic lupus erythematosus, systemic sclerosis, primary sjogren's syndrome, and so on. Connective tissue diseases (CTDs) is a widely immune-mediated heterogeneous disease. The molecular mechanism of miRNA-155 in different CTDs is complex and changeable. In this paper, we systematically review the correlation between the miRNA-155 and CTDs, and demonstrated the molecular mechanism of miRNA-155 in connective tissue diseases. It might bring new potential markers for the diagnosis and treatment.
\end{abstract}

\section{Keywords}

miRNA-155, connective tissue diseases, diagnosis, treatment

\section{Introduction}

Connective tissue diseases (CTDs) are widely immune-mediated heterogeneous diseases, which are characterized by the loss of autoimmune response regulation, complement activation, interferon regulatory dysfunction and related inflammatory infiltration. Because the whole body is covered with connective tissue, the clinical manifestations of CTDs are various, which can involve skin, mucous membrane, blood vessels, joint muscles and various organ systems of the whole body. At present, the cause of the disease is not clear. Through the cross analysis of the genome related research of autoimmune diseases [1] and the common regulatory mechanism of autoimmune diseases [2], these diseases have significant genetic tendency. Besides, drugs, gender and environment also play an important role in the occurrence and development of the disease. According to the criteria of rheumatism classification formulated by American rheumatic Association in 1983, CTDs include rheumatoid arthritis, systemic lupus erythematosus, sjogren's syndrome, systemic sclerosis, idiopathic inflammatory disease, Behcet's disease and vasculitis.

MicroRNA (miRNAs) is a single strand non-coding RNA sequence, which is involved in the regulation of gene expression at the post transcription level in animals and plants [3]. MiRNA and its target gene mRNA sequence accelerate mRNA degradation or inhibit mRNA translation through complete or nearly complete complementary binding, thus negatively regulate gene expression [4-5]. The morphological feature of miRNA gene is the structure of stem ring precursor RNA, one (sometimes two) stem chain is the source of mature miRNA [6]. From the gene level analysis, DNA is transcribed into pri-miRNA by RNA polymerase II [7]. Pri-miRNA is usually modified by splicing, capping and polyadenylation in a way similar to protein coding mRNA [8], and dgcr8 is responsible for identifying Pri-miRNA in the nucleus I-mirna stem ring structure, then recruit Drosha endonuclease to cut and release stem ring precursor, stem ring precursor enters the cytoplasm, and endonuclease Dicer cuts the ring area of the precursor, releasing mature miRNA [9-10]. More and more literatures have confirmed that two mature RNA sequences formed by miRNA gene expression have biological functions [4] [11]. About $30 \%$ of human genes are regulated by miRNA, and also participate in metabolism, growth and other 
pathophysiological processes [4].

The abnormal expression of miRNAs is related to many diseases. Because of its targeted interaction and its effect on diseases, more and more researchers pay attention to the causal relationship of disease development, which reflects the application value in clinical evaluation. Non-coding microRNA-155 (miRNA-155) is an important member of the miRNAs family, which consists of 23 nucleotides. It is encoded by miR-155 host gene (MiR155HG) located on chromosome 21 [12]. MiRNA-155 has been recognized as an important regulator of immune cell development and immune response. MiR-155HG was matured by the sequential processing of miR155HG transcripts in 3 'or 5' terminals by Drosha and Dicer endonucleases. Then, miRNA-155-5p or miRNA-155-3p can control gene expression by binding to the 3 'untranslated (UTR) coding region in the target mRNA [13]. Previous evidence shows that miRNA-155 is differentially expressed in patients with CTDs, which leads to the abnormal production of autoantibodies and pro-inflammatory cytokines and participates in the pathogenesis of CTDs. The regulatory mechanism of miRNA -155 in different CTDs is complex and changeable, which makes this paper systematically review the correlation between mirna-155 and CTDs.

\section{MiRNA-155 plays a role in immune cells}

T cells, B cells, MC cells, NK cells and DC cells in patients with autoimmune diseases show unique properties and play an important role in disease progression and pathology. The pathogenic autoantibodies and cytokines secreted by these immune cells participate in inflammation and autoimmune response. In this review, we demonstrate the latest development of miRNA-155 in immune cells. In particular, we concentrate on regulatory mechanism and the pathogenic immune response. These findings are essential for understanding the nature of miRNA-155 in the pathogenesis of various autoimmune diseases.

\subsection{The role of miRNA-155 in B cells}

Maladjusted B cell development led to a significant accumulation of preB cells in mice, and miRNA-155 was used to induce polyclonal expansion, which led to B-cell lymphoma in mice. It is suggested that mirna-155 may play an important role in B cell function [14]. Follicles and germinal centers in miRNA-155-deficient B cells decreased and the ability of these B cells to produce IgG1 antibody with high affinity decreased significantly [15]. It is reported that the lack of inositol phosphatase (SHIP1) in B lymphocytes leads to the formation of germinal centers and the conversion of antibody types [16]. O'Connell [17] et al also pointed out that SHIP1 is the direct target of miRNA-155 in hematopoietic system, and mirna-155 directly interacts with 3'UTR to inhibit the production of SHIP1. In conclusion, these results suggest that miRNA-155- SHIP1 axis may play an important role in regulating $\mathrm{B}$ cell activation and proliferation.

\subsection{MiRNA -155 participates in Th cell differentiation}

It was shown that the down-regulation of mirna-155 resulted in the increase of Th1 and Th17 cells, but enhanced the production of Th2 cytokines [18]. These findings were confirmed again by Blüml et al. [19], who studied the cytokine of Th1 and Th2 polarization and found the trend of increasing IL-4 level, but there was no significant difference in IFN - $\gamma$ expression in miRNA-155 deficient mice. In another study, it was found that IFN - $\gamma$ receptor $\alpha$ chain is another target of miRNA-155 in Th1 cells. MiRNA-155 promotes the differentiation of CD4 + T cells into Th1 cells by inhibiting IFN $-\gamma$ signal pathway [20]. It may be that miRNA-155 can reduce the expression of SOCS1 in activated CD4 + T cells in some autoimmune diseases, thus activating IL-2 / STAT5 and IL-6/STAT3 signaling pathways, inducing Treg/Th17 cell differentiation and enhancing Th17 function [21]. In conclusion, miRNA-155 can prompte CD4 + T cells differentiate into different cells and play different roles in immune.

\subsection{The role of miRNA155 in Treg cells}

Regulatory $\mathrm{T}$ (Treg) cells are essential for maintaining immune tolerance. At present, many studies have shown that miRNA-155 can promote the development and proliferation of Treg cells. For example, Foxp3 is a transcription factor necessary for the normal development of Treg cells, and miRNA-155 is a direct target of Foxp3. Through the analysis of Foxp3 regulatory genes, the number of Treg cells in thymus and peripheral blood of miRNA-155 deficient mice model is significantly reduced, so miRNA-155 promotes the development of Treg. As is known to all, IL-2 is very important in Treg cell homeostasis, and SOCS1 is the direct target of miRNA-155. Lu et al. [22] found that Treg cells with miRNA-155 deficiency have decreased IL-2 signal transduction ability, while the absence of miRNA-155 in Treg cells resulted in increased expression of SOCS1, so the stable state of 
Treg cells could be maintained by inhibiting the expression of mirna- 155 to regulate the expression level of SOCS1. Therefore, miRNA-155 regulates Treg cells to play immune effects on maintaining immune homeostasis.

\subsection{The profound effect of miRNA-155 on DC immune response}

With the research of dendritic cells (DC), miRNA-155 has been found to have a profound impact on the immune response of DC. MiRNA-155 gene affects the synthesis of interferon (IFN) type I in plasma like dendritic cells by interfering with the intracellular signaling pathway downstream of Toll like receptors 7 and 9 (TLR-7, TLR-9) [23]. Ceppi et al. [24] found that inflammation can promote the differentiation and maturation of DC cells. The Toll like receptor/interleukin-1 (TLR/IL-1) inflammatory pathway is the target of miRNA-155, and the up-regulation of mirna-155 promotes the maturation of DC. Therefore, miRNA-155 is a part of negative feedback loop in mature human dendritic cells, which can down regulate inflammatory cytokines by the stimulation of microorganisms generation.

In conclusion, these data indicate that miRNA-155 plays different roles in different immune cells

\section{MiRNA-155 and rheumatoid arthritis}

Rheumatoid arthritis (RA) is a kind of systemic inflammatory autoimmune disease, which mainly involves the joints, and extraarticular manifestations are also included in it, such as vasculitis, interstitial lung disease, and so on. It is characterized by irreversible chronic destructive arthritis. Besides, $1 \%$ of the world's population suffer from RA [25]. So far, the pathogenesis of RA has not been demonstrated clearly. Early diagnosis and early treatment can improve the prognosis and prevent RA patients from serious disease. Therefore, more biomarkers are needed to identify for the diagnosis of diseases in early stage.

The differential expression of miRNA-155 in RA indicates its important role in the natural process of RA. Previous evidence showed that overexpression of mirna-155 in peripheral blood CD14 + monocytes of RA patients could promote the production of cytokines and chemokines related to RA synovitis, such as TNF - $\alpha$, IL-6, IL-1 $\beta$, IL-8, chemokine ligand (CCL) 3, CCl4, CCL5, CCL8, but reduce the production of IL-10, C-C chemokine receptor type 2 (CCR2) [26]. One of the important targets of miRNA-155 is SOCS1, which can promote expression of TNF and IL-1 by reducing the expression of SOCS1 [27]. These abnormal expressions of miRNA-155 may be involved in the pathogenesis of RA, and may also be an effective indicator for the diagnosis and monitoring of RA disease activity.

\section{MiRNA-155 and systemic lupus erythematosus}

Systemic lupus erythematosus (SLE) is a kind of autoimmune disease that occurs frequently in women of childbearing age. Systemic lupus erythematosus has a wide range of clinical manifestations, but its course of disease and organ involvement are unpredictable. It is characterized by the abnormal response of the autoimmune system to the autoantigen, which can produce a large number of abnormal serum antibodies, and can have a substantial impact on any organ and tissue [28]. The pathogenesis of SLE is complex and changeable. Recent studies have shown that internal and external factors lead to abnormal expression of miRNAs in SLE. It plays a pathogenic role in different stages of cell activity and widely participates in many aspects of the pathogenesis of SLE. In particular, the expression of miRNA-155 may be a new target for SLE.

As reported, abnormal expression of miRNA-155 was found in T cells, B cells, plasma, renal tubular epithelial cells, mesangial cells and podocytes in peripheral blood of SLE patients. MirRNA-155 was up-regulated in PBMC of patients with severe active SLE, especially in lupus nephritis (LN) and hematological abnormalities, which indicated that miRNA-155 was related to disease progression and target organ damage [29]. In terms of pathogenesis, miRNA-155-Peli1-c-Rel pathway can regulate the differentiation of Tfh cells [30], which is related to the disease activity of SLE and plays an important role in the pathogenesis of SLE.

In conclusion, miRNAs-155 might be potential new biomarkers of SLE and help to elucidate the pathogenesis of LN. Based on studies of SLE miRNA-155, it is shown that the characteristics of miRNA-155 will provide new biomarkers for the diagnosis of SLE and the evaluation of LN.

\section{MiRNA-155 and systemic sclerosis}

Systemic sclerosis (SSC) is a chronic multisystem disease with unknown etiology, which is characterized by vascular damage, immune system activation and diffuse tissue fibrosis. In recent years, miRNAs have been considered as a potential key factor in the pathogenesis of SSC [31]. It has been confirmed that miRNA-155 and other miRNAs are differentially expressed in SSC patients, but the pathogenesis still needs further investigation. 
MiRNA155 is a kind of microRNAs that has been studied more in CTDs. The expression of miRNA-155 in SSC lung fibroblasts depends on the up-regulated activation of inflammatory corpuscles. The inhibition of signal pathway of inflammatory corpuscles down-regulatethe expressionof miRNA-155. The inflammatory body mediated collagen synthesis could not be induced with miRNA-155. When miRNA-155 is expressed in the fibroblasts lacking miRNA-155, collagen synthesis can be restored, so miRNA -155 is the key miRNA driving fibrosis [32]. MiRNAs in SSC patients can reflect the pathogenic state of autoimmunity, which indicates that they play an important role in the pathogenesis of the disease.

\section{MiRNA-155 and primary Sjogren's syndrome}

Primary Sjogren's syndrome (PSS) is a common chronic inflammatory autoimmune disease. Its main characteristics are local lymphocytic infiltration and progressive damage of exocrine glands, as well as the production of autoantibodies (anti Ro/SSA and anti LA/SSB) and rheumatoid factors induced by the dysfunction of the immune system. The main symptoms of pSS are keratoconjunctivitis sicca and xerostomia. It can also involve multiple organs of the whole body, such as renal tubular acidosis and interstitial lung disease [33]. There will be an increase or decrease of specific miRNAs in circulating blood and blood cells, labial gland and eye tissue. These miRNAs play a key role in regulating inflammation, delaying or promoting wound healing, cell differentiation, metabolism and survival.

In recent years, there are some studies on the mechanism of miRNAs on the pathogenesis of PSS. It was found that many microRNAs were expressed in many target organs of PSS, such as miRNA-181a and miRNA-16 in salivary gland, miRNA-223 and miRNA-146a/B in peripheral blood mononuclear cells, and miRNA-17-29 in small salivary gland [34]. According to a study on the expression of miRNA-155 in PBMCs of patients with PSS, it indicated that the relative expression of miRNA-155 in patients with PSS was increased (2.5 times of that in the control group) [35]. What's more, the expression level of miRNA-155 was higher than that of the control. Overexpressed miRNA-155 is associated with clinical features of the disease, such as more pronounced dry eye symptoms [36]. The mechanism of miRNAs may be related to traditional biomarkers such as PSS, RO (SSA), La (SSB) [37]. Therefore, it indicates the potential significance of miRNAs in pSS patients.

\section{Conclusion and Prospect}

Connective tissue disease is a complex disease caused by environmental, genetic, and immune mechanisms. Although a variety of antibodies have been used in the clinical diagnosis of diseases, due to the low specificity and sensitivity, the goal of clinical diagnosis has not been achieved. For patients on early stage with poor recognition, they cannot be diagnosed and treated in time. miRNA-155 is expected to be a new marker in diagnosis and recognition of connective tissue disease. At present, the evidence shows that miRNA-155 plays an important role in some key steps of the pathogenesis of CTDs. However, the exact mechanism of miRNA-155 is not clear. Further investigation is needed for further work in clinic.

\section{References}

[1] Farh KK, Marson A, Zhu J, et al. (2015). Genetic and epigenetic fine mapping of causal autoimmune disease variants [J]. Nature, 518(7539): 337-343.

[2] Shooshtari P, Huang H, Cotsapas C. (2017). Integrative Genetic and Epigenetic Analysis Uncovers Regulatory Mechanisms of Autoimmune Disease [J]. Am J Hum Genet, 101(1): 75-86.

[3] Huntzinger E, Izaurralde E. (2011). Gene silencing by microRNAs: contributions of translational repression and mRNA decay [J]. Nat Rev Genet, 12(2): 99-110.

[4] Bartel DP. (2004). MicroRNAs: genomics, biogenesis, mechanism, and function [J]. Cell, 116(2): 281-297.

[5] Roberts TC. (2015). The microRNA machinery [J]. Adv Exp Med Biol, 887: 15-30.

[6] Lee RC, Ambros V. (2001). An extensive class of small RNAs in Caenorhabditis elegans [J]. Science, 294(5543): 862-864.

[7] Bortolin-Cavaille ML, Dance M, Weber M, et al. (2009). C19MC microRNAs are processed from introns of large Pol-II, non-protein-coding transcripts [J]. Nucleic Acids Res, 37(10): 3464-3473.

[8] Cai X, Hagedorn CH, Cullen BR. (2004). Human microRNAs are processed from capped, polyadenylated transcripts that can also function as mRNAs [J]. RNA, 10(12): 1957-1966.

[9] Ballarino M, Pagano F, Girardi E, et al. (2009). Coupled RNA processing and transcription of intergenic primary microRNAs [J]. Mol Cell Biol, 29(20): 5632-5638.

[10] Han J, Lee Y, Yeom KH, et al. (2004). The Drosha-DGCR8 complex in primary microRNA processing [J]. Genes Dev, 18(24): 
3016-3027.

[11] Baulina NM, Kulakova OG, Favorova OO. (2016). MicroRNAs: the role in autoimmune inflammation [J]. Acta Naturae, 8(1): 21-33.

[12] Tam W. (2001). Identification and characterization of human BIC, a gene on chromosome 21 that encodes a noncoding RNA [J]. Gene, 22: 274.

[13] Wu XY, Pais EM, Li Lan, et al. (2017). MicroRNA-155: a Novel Armamentarium Against Inflammatory Diseases [J]. Inflammation, 40(2): 708-716.

[14] Costinean S, Zanesi N, Pekarsky Y, et al. (2006). Pre-B cell proliferation and lymphoblastic leukemia/high-grade lymphoma in E(mu)-miR155 transgenic mice [J]. Proc Natl Acad Sci USA, 103(18): 7024-7029.

[15] Vigorito E, Perks KL, Abreu-Goodger C, et al. (2007). microRNA-155 regulates the generation of immunoglobulin class-switched plasma cells [J]. Immunity, 27(6): 847-859.

[16] Leung WH, Tarasenko T, Bolland S. (2009). Differential roles for the inositol phosphatase SHIP in the regulation of macrophages and lymphocytes [J]. Immunol Res, 43(1-3): 243-251.

[17] O’Connell RM, Chaudhuri AA, Rao DS, et al. (2009). Inositol phosphatase SHIP1 is a primary target of miR-155 [J]. Proc Natl Acad Sci USA, 106(17): 7113-7118.

[18] Zhang J, Cheng Y, Cui W, et al. (2014). MicroRNA-155 modulates Th1 and Th17 cell differentiation and is associated with multiple sclerosis and experimental autoimmune encephalomyelitis [J]. J Neuroimmunol, 266(1-2), 56-63.

[19] Blüml S, Bonelli M, Niederreiter B, et al. (2011). Essential role for micro-RNA 155 in the pathogenesis of autoimmune arthritis in mice [J]. Arthritis Rheum, 63(5): 1281-1288.

[20] Banerjee A, Schambach F, DeJong CS, et al. (2010). Micro-RNA-155 inhibits IFN-gamma signaling in CD4+ T cells [J]. Eur J Immunol, 40(1): 225-231.

[21] Escobar T, Yu CR, Muljo SA, et al. (2013). STAT3 activates miR-155 in Th17 cells and acts in concert to promote experimental autoimmune uveitis [J]. Invest Ophthalmol Vis Sci, 54(6): 4017-4025.

[22] Lu LF, Thai TH, Calado DP, et al. (2009). Foxp3-dependent microRNA155 confers competitive fitness to regulatory T cells by targeting SOCS1 protein [J]. Immunity, 30(1): 80-91.

[23] Banerjee A, Schambach F, DeJong CS, et al. (2010). MicroRNAs in the key events of systemic lupus erythematosus pathogenesis [J]. Eur J Immunol, 40(1): 225-231.

[24] Ceppi M, Pereira PM, Dunand-Sauthier I, et al. (2009). MicroRNA-155 modulates the interleukin-1 signaling pathway in activated human monocyte-derived dendritic cells [J]. Proc Natl Acad Sci USA, 106(8): 2735-2740.

[25] Frisell T, Hellgren K, Alfredsson L, et al. (2016). Familial aggregation of arthritis-related diseases in seropositive and seronegative rheumatoid arthritis: a register-based case-control study in Sweden [J]. Ann Rheum Dis, 75(1): 183-189.

[26] Elmesmari A, Fraser AR, Wood C et al. (2016). MicroRNA-155 regulates monocyte chemokine and chemokine receptor expression in Rheumatoid Arthritis [J]. Rheumatology (Oxford), 55(11): 2056-2065.

[27] Li X, Tian F, Wang F. (2013). Rheumatoid arthritis-associated microRNA-155 targets SOCS1 and upregulates TNF-a and IL-1b in PBMCs [J]. Int J Mol Sci, 14(12): 23910-23921.

[28] Cojocaru M, Cojocaru IM, Silosi I, et al. (2011). Manifestations of systemic lupus erythematosus [J]. Maedica (Buchar), 6(4): 330-336.

[29] Zhang H, Huang X, Ye L, et al. (2018). B Cell-Related Circulating MicroRNAs With the Potential Value of Biomarkers in the Differential Diagnosis, and Distinguishment Between the Disease Activity and Lupus Nephritis for Systemic Lupus Erythematosus [J]. Front Immunol, 9: 1473.

[30] Liu WH, Kang SG, Huang Z, et al. (2016). A miR-155-Peli1-c-Rel pathway controls the generation and function of T follicular helper cells [J]. J Exp Med, 213(9): 1901-1919.

[31] Broen JC, Radstake TR, Rossato M. (2014). The role of genetics and epigenetics in the pathogenesis of systemic sclerosis [J]. Nat Rev Rheumatol, 10(11): 671-681.

[32] Artlett CM, Sassi-Gaha S, Hope JL, et al. (2017). Mir-155 is overexpressed in systemic sclerosis fibroblasts and is required for NLRP3 inflammasome mediated collagen synthesis during fibrosis [J]. Arthritis Res Ther, 19(1): 144.

[33] Nair JJ, Singh TP. (2017). Sjögren's syndrome: review of the aetiology, pathophysiology \& potential therapeutic interventions [J]. J Clin Exp Dent, 9(4): e584-e589.

[34] Reale M, D’Angelo C, Costantini E, et al. (2018). MicroRNA in Sjögren’ssyndrome: Their potential roles in pathogenesis and diagnosis [J]. J Immunol Res, 2018: 7510174.

[35] Pauley KM, Stewart CM, Gauna AE, et al. (2011). Altered miR-146a expression in Sjögren's syndrome and its functional role in innate immunity [J]. Eur J Immunol, 41(7): 2029-2039.

[36] Shi H, Zheng LY, Zhang P, et al. (2014). miR-146a and miR-155 expression in PBMCs from patients with Sjogren's syndrome [J]. J Oral Pathol Med, 43(10): 792-797. 
[37] Liang CY, Xiong K, Szulwach KE, et al. Sjogren syndrome antigen B (SSB)/La promotes global microRNA expression by binding microRNA precursors through stem-loop recognition [J]. J Biol Chem, 2013, 288(1): 723-736. 